\title{
Correction to: Rings, Modules, and Closure Operations
}

\section{Correction to:}

\section{J. Elliott, Rings, Modules, and Closure Operations, Undergraduate Topics in Computer Science, https://doi.org/10.1007/978-3-030-24401-9}

In the original version of the book, the author missed to provide corrections in most of the chapters. The book now has been updated with all the corrections.

\section{Replacement in the Proposition:}

Statements (6) and (7) of Proposition 2.8.7 has been corrected with the following in page 132:

(6) If I is regular, then $I^{p}=\bigcap\left\{x R: x \in T(R)^{\mathrm{reg}}, x R \supseteq I\right\}$ is principal if and only if $\operatorname{gcd}(c S)$ exists for all regular $c \in R$. If these conditions hold, then one has $I^{p}=(\operatorname{gcd}(S))$ and $\operatorname{gcd}(c S)=c \operatorname{gcd}(S)$ for all regular $c \in R$.

(7) If $I$ is regular, then $I^{v}$ is principal if and only if $I^{v}=I^{p}$ and $I^{p}$ is principal, if and only if $I^{v}=I^{p}$ and $\operatorname{gcd}(d S)$ exists for all regular $d \in R$.

Statements (1)-(7) of Proposition 2.8.19 has been corrected with the following in page 135:

(1) $R$ is a weak GCD ring and $I^{p}=I^{v}$ for every ideal I of $R$ generated by finitely many regular elements of $R$.

(2) $I^{p}$ is principal and $I^{p}=I^{v}$ for every ideal I of $R$ generated by finitely many regular elements of $R$.

(3) $I^{v}$ is principal for every ideal $I$ of $R$ generated by finitely many regular elements of $R$.

The updated version of the book can be found at https://doi.org/10.1007/978-3-030-24401-9 
(4) $\operatorname{gcd}(a, b)$ exists and $(a, b)^{v}=(a, b)^{p}$ for all regular $a, b \in R$ (in which case $\operatorname{gcd}(c a, c b)=c \operatorname{gcd}(a, b)$ for all regular $c \in R)$.

(5) $(a, b)^{v}$ is principal for all regular $a, b \in R$.

(6) The intersection of any two regular principal ideals of $R$ is principal.

(7) The lcm of any two regular elements of $R$ exists.

Proposition 2.10.8 has been replaced with the following in page 148.

Proposition 2.10.8. Let $R$ be a ring, and let be a unital generalized semistar operation on $R$.

(1) $R$ is GCD if and only if $R$ is p-Bézout.

(2) $R$ is strong GCD if and only if $R$ is a p-PIR.

(3) $R$ is weak GCD if and only if $R$ is weak p-Bézout.

(4) $R$ is weak strong GCD if and only if $R$ is a weak p-PIR.

(5) $R$ is $\star$-Bézout if and only if $R$ is a GCD ring such that $\star_{t} \simeq p_{t}$.

(6) $R$ is $a \star$-PIR if and only if $R$ is a strong GCD ring such that $\star \simeq p$.

(7) $R$ is $\star$-Bézout if and only if $R$ is a weak GCD ring such that $\star_{t} \simeq_{\operatorname{rg}} p_{t}$.

(8) $R$ is a weak $\star$-PIR if and only if $R$ is a weak strong GCD ring such that $\star \simeq$ rg $p$.

Statement (6) of Proposition 2.10.20 has been corrected with the following in page 151 .

(6) $R$ is weak GCD ring and $\star_{t} \simeq_{\mathrm{wk}} p_{t}\left(\right.$ or $\left.\star_{t} \simeq_{\mathrm{wk}} t \simeq_{\mathrm{wk}} p_{t}\right)$.

\section{Replacement in the Lemma:}

Statement (2) of Lemma 2.8.18 has been corrected with the following in page 135.

(2) $(a, b)^{v}=(a, b)^{p}$ and $\operatorname{gcd}(c a, c b)$ exists for all regular $c \in R$ (in which case $\operatorname{gcd}(c a, c b)=c \operatorname{gcd}(a, b))$.

\section{Replacement in the Theorem:}

Theorem 2.10.16 has been replaced with the following in page 150 .

Theorem 2.10.16. Let $R$ be a ring. The following conditions are equivalent.

(1) $R$ is factorial.

(2) $R$ is a weak $p_{t}-P I R$, that is, $I^{p_{t}}$ is principal for all regularly generated ideals I of $R$.

(3) $R$ is a weak $p$-PIR and $p \simeq_{\mathrm{rg}} p_{t}$.

(4) $R$ is a weak strong $G C D$ ring and $p \simeq_{\mathrm{rg}} p_{t}$.

(5) $R$ is a weak GCD ring and satisfies ACCRP.

(6) $R$ is an r-atomic weak GCD ring. 
Moreover, each of the equivalent conditions above in conjunction with the condition that $p \simeq_{\mathrm{rg}} v\left(\right.$ or $\left.p_{t} \simeq_{\mathrm{rg}} t\right)$ is equivalent to each of the following conditions, all of which are equivalent.

(7) $R$ is factorial and $p \simeq_{\mathrm{rg}} v$.

(8) $R$ is a weak $t$-PIR, that is, $I^{t}$ is principal for all regularly generated ideals I of $R$.

(9) $R$ is a weak Mori weak v-PIR.

(10) $R$ is a weak $T V$ weak $v$-PIR.

(11) $R$ is a weak Krull ring $R$ with $\mathrm{Cl}_{\mathrm{wk}}^{t}(R)=0$.

\section{Replacement in Corrolary:}

Statement (4) of Corollary 2.10.21 has been corrected with the following in page 151.

(4) $R$ is a weak $G C D$ ring and $p_{t} \simeq_{\mathrm{wk}} t$.

\section{Corrections in Exercises:}

Part (b) of Exercise 13 has been deleted (and relabelled the remaining parts) in page 177.

In Exercise 10. "Use Exercise 2.7.13" has been changed into "Use Exercise 2.7.14" in page 180 .

"(See Exercise 3.7.6.)" has been changed into "(See Exercise 3.7.7.)" in page 229. "(See also Exercise 3.7.12.)" has been changed into "(See also Exercise 3.7.13.)" in page 230 .

In Exercise 15. "totally ordered group" has been changed into "totally ordered abelian group" in page 277.

Exercise 25. "Exercise 2.7.13" has been changed into "Exercise 2.7.14" in page 278.

Exercise 16. "(See Exercise 3.3.10.)" has been changed into "(See Exercise 3.4.10.)" in page 283.

Exercise 8. "Use Corollary 3.8.5" has been changed into "Use Proposition 3.8.3" in page 284 .

Exercise 7. "Exercise 2.7.13" has been changed into "Exercise 2.7.14". "Exercise 2.7.18" has been changed into "Exercise 2.7.19" in page 288 .

Exercise 10. "Exercise 3.2.3.11" has been changed into "Exercise 3.2.11" in page 288.

Exercise 17. "Exercise 3.6.22" has been changed into "Exercise 3.6.25" in page 290.

Exercise 9. "Exercise 2.7.1" has been changed into "Exercise 2.7.18" in page 291. Exerise 29(e). "Use part (d) to complete Exercise 24" has been changed into "Use part (d) to complete Exercise 28" in page 358. 